\title{
Magnetic entropy landscape and Grüneisen parameter of a quantum spin ladder
}

\author{
H. Ryll, ${ }^{1}$ K. Kiefer, ${ }^{1}$ Ch. Rüegg, ${ }^{2,3,4}$ S. Ward, ${ }^{2,3}$ K. W. Krämer, ${ }^{5}$ D. Biner, ${ }^{5}$ P. Bouillot, ${ }^{6,7}$ E. Coira, ${ }^{4}$ \\ T. Giamarchi, ${ }^{4}$ and C. Kollath ${ }^{8}$ \\ ${ }^{1}$ Helmholtz Center Berlin for Materials and Energy, D-14109 Berlin, Germany \\ ${ }^{2}$ Laboratory for Neutron Scattering, Paul Scherrer Institute, CH-5232 Villigen PSI, Switzerland \\ ${ }^{3}$ London Centre for Nanotechnology and Department of Physics and Astronomy, University College London, \\ London WC1E 6BT, England, United Kingdom \\ ${ }^{4}$ Département de Physique de la Matière Condensée, MaNEP, University of Geneva, CH-1211 Geneva, Switzerland \\ ${ }^{5}$ Department of Chemistry and Biochemistry, University of Bern, CH-3012 Bern, Switzerland \\ ${ }^{6}$ Department of Medical Imaging and Information Sciences, Interventional Neuroradiology Unit, \\ University Hospitals of Geneva, CH-1211 Geneva, Switzerland \\ ${ }^{7}$ Laboratory for Hydraulic Machines, École Polytechnique Fédérale de Lausanne, CH-1015 Lausanne, Switzerland \\ ${ }^{8}$ Helmholtz-Institut für Strahlen und Kernphysik, University of Bonn, Nussallee 14-16, D-53115 Bonn, Germany
}

(Received 16 November 2013; revised manuscript received 1 April 2014; published 18 April 2014)

\begin{abstract}
We present measurements of the magnetic entropy landscape and Grüneisen parameter of the $\mathrm{Cu}^{2+}$ complex $\left(\mathrm{C}_{5} \mathrm{H}_{12} \mathrm{~N}\right)_{2} \mathrm{CuCl}_{4}$ in a magnetic field. Our thermodynamic measurements are in very good agreement with a theoretical description by a $S=1 / 2$ Heisenberg ladder model. Due to its excellent experimental access, the compound crosses two quantum critical points in the applied range of the magnetic field, first from a gapped unpolarized state to a critical phase and then to a gapped fully polarized state. This behavior is reflected directly in the magnetic entropy map. Due to the remarkable properties of the magnetic Grüneisen parameter, we are able to discuss the validity of critical behavior with respect to temperature and magnetic field for this model quasi-one-dimensional system.
\end{abstract}

DOI: 10.1103/PhysRevB.89.144416

PACS number(s): 75.10.Jm, 75.40.Cx, 75.40.Mg

\section{INTRODUCTION}

Quantum phase transitions (QPTs) are of central importance in condensed-matter physics [1]. Although limited to the experimentally inaccessible temperature of $0 \mathrm{~K}$, quantum critical points (QCPs) influence large areas of the low-temperature phase diagram [2-5]. Thermodynamic and magnetic properties are very well suited to reveal the temperature and magnetic field range, for which critical behavior applies.

In this paper we present measurements of both the magnetic entropy and Grüneisen parameter of the magnetic insulator $\left(\mathrm{C}_{5} \mathrm{H}_{12} \mathrm{~N}\right)_{2} \mathrm{CuCl}_{4}$ [6]. Previously such measurements were conducted for metallic quantum critical systems, such as the heavy fermion compound $\mathrm{YbRh}_{2} \mathrm{Si}_{2}[3,4]$ and the strongly correlated electron system $\mathrm{Sr}_{3} \mathrm{Ru}_{2} \mathrm{O}_{7}$ [5]. As predicted by theory, a map of the entropy landscape can identify different quantum phases [5] and the occurring extrema quantum critical points [7]. The Grüneisen parameter signals a critical point by its divergence. Furthermore, the temperature or the magnetic field dependence of the Grüneisen parameter gives a very direct way to extract the critical exponents of QCPs. In contrast to the previously mentioned materials, magnetic insulators have the advantage that the Hamiltonian and all of its parameters can be determined. Hence a fully quantitative comparison between experiment and theory is possible.

Of special interest are one-dimensional systems, due to their remarkable physics and properties [8]. A particularly interesting case is the two-leg antiferromagnetic $S=1 / 2$ Heisenberg ladder. An external magnetic field drives the spin ladder through two QPTs. Upon increasing field, the magnetic system changes from a gapped quantum disordered spin liquid (QD) with magnon excitations, to a gapless spin Luttinger liquid (LL) with spinon excitations, to a fully polarized state, that is once more gapped [9,11,12]. This behavior has been observed, for instance, for the spin ladder compound $\left(\mathrm{C}_{5} \mathrm{H}_{12} \mathrm{~N}\right)_{2} \mathrm{CuBr}_{4}$ [9-11,13-16]. Unfortunately the exchange couplings of $\left(\mathrm{C}_{5} \mathrm{H}_{12} \mathrm{~N}\right)_{2} \mathrm{CuBr}_{4}$ make it difficult to access experimentally the upper QCP, and no data of entropy or Grüneisen parameter is available. However, the investigation of the two different QCPs by magnetic entropy and Grüneisen parameter would be very interesting in order to compare the properties and symmetries of the two QCPs. Fortunately the chemical flexibility of metal-complex magnetic insulators allows chemical control of the exchange parameters. In the present case, replacing $\mathrm{Br}$ with $\mathrm{Cl}$ reduces the values of the exchange parameters and thereby the critical magnetic fields [6,17], which makes both QCPs accessible in experiments.

\section{EXPERIMENTAL RESULTS AND THEORETICAL MODELING}

\section{A. Experimental setup}

In order to obtain the magnetic entropy and Grüneisen parameter we performed measurements of the magnetization, the heat capacity, and the magnetocaloric effect (MCE). These measurements were carried out at the Laboratory for Magnetic Measurements at the Helmholtz Center Berlin. For magnetization measurements down to $100 \mathrm{mK}$ a Faraday force magnetometer with gradient coil was used [18], with magnetic fields varying between 0 and $6 \mathrm{~T}$. Heat capacity and MCE were measured between $300 \mathrm{mK}$ and $3 \mathrm{~K}$, in magnetic fields up to $12 \mathrm{~T}$, using the same single-crystal sample of mass $3.71 \mathrm{mg}$. The MCE was measured quasi-isothermally, meaning the amount of heat $\delta Q$ that is generated or absorbed 

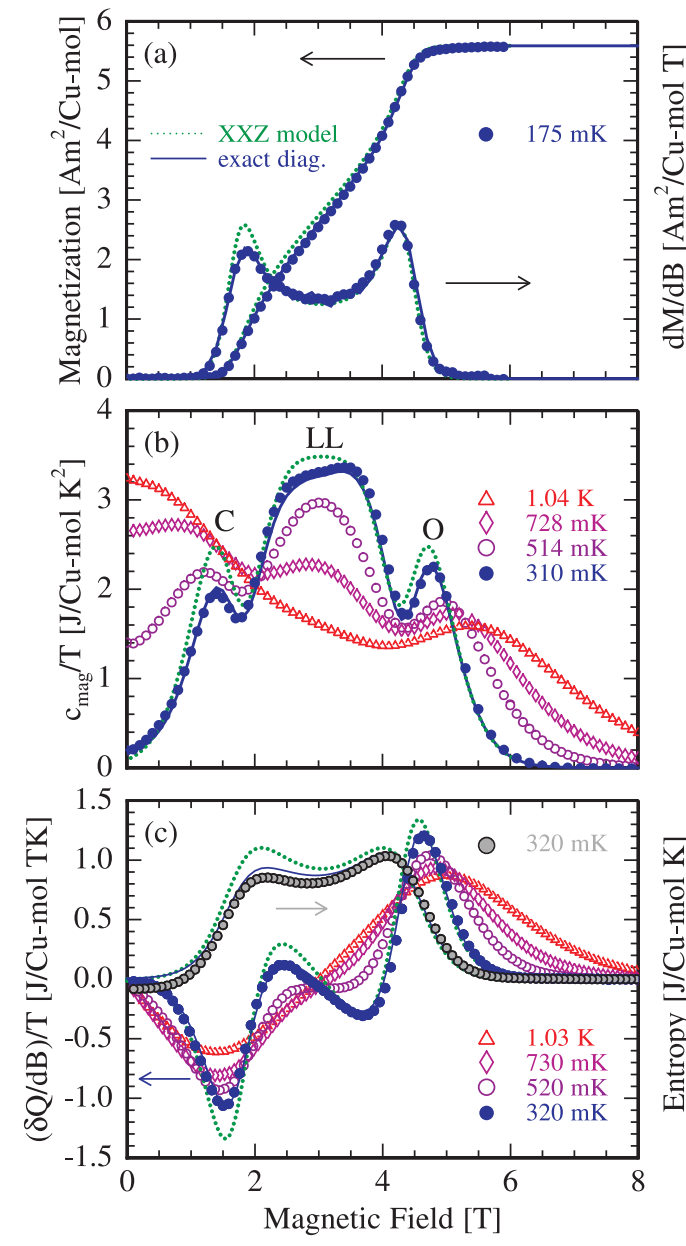

FIG. 1. (Color online) Magnetization (a), heat capacity (b), and magnetocaloric effect measurements (c) of $\left(\mathrm{C}_{5} \mathrm{H}_{12} \mathrm{~N}\right)_{2} \mathrm{CuCl}_{4}$ (points) with comparison to calculations (blue line, exact diagonalization 10 rungs; dashed green line, $\mathrm{XXZ}$ model). $\mathrm{C}$ and $\mathrm{O}$ mark the closing and reopening of the spin gap, and LL marks the spin Luttinger liquid. Quantitative entropy data are obtained from the MCE (gray points in lower panel); see text.

by the sample for a changing field $d B$ was measured. For all measurements, the magnetic field was aligned parallel to the crystallographic $a$ axis, parallel to the ladder direction. The samples were high-quality single crystals, grown at the University of Bern by slow evaporation of an oversaturated solution [19].

\section{B. Magnetization, heat capacity, and magnetocaloric effect}

In Fig. 1 we present the experimental data. Figure 1(a) shows an exemplary magnetization measurement at $T=$ $175 \mathrm{mK}$ and the derivative of magnetization with respect to the magnetic field $d M / d B$. Qualitatively, those measurements confirm the expected ladder behavior [13]: there is no magnetization below the lower critical field $B_{c 1}=1.73(6) \mathrm{T}$, due to the spin gap. The increase of magnetization above $B_{c 1}$ follows a square-root behavior, until an inflection point is reached at half magnetization. Above the upper critical field
$B_{c 2}=4.38(8) \mathrm{T}$ the spins are fully polarized and saturation is reached.

Figure 1(b) shows the magnetic contribution of heat capacity at constant temperature. Further temperature scans between 0.3 and $30 \mathrm{~K}$ and up to full magnetic saturation at $12 \mathrm{~T}$ were used for an accurate determination of the lattice heat capacity $[9,17]$, which was subtracted for the plot. The data were further divided by temperature and show two peaks, that mark the closing and reopening of the spin gap. Extrapolations of these peaks to $0 \mathrm{~K}$ yield $B_{c 1}$ and $B_{c 2}$, in good agreement with magnetization results. Between those peaks is a region with high magnetic heat capacity, which has its origin in the continuous spinon excitations of a spin Luttinger liquid. Above a temperature of $1 \mathrm{~K}$, which is comparable in energy to the spin-exchange couplings, this pronounced structure disappears.

Figure 1(c) shows quantitative MCE measurements. The MCE is linked to the magnetization $M$ via the Maxwell relation $(1 / \mathrm{T}) /(\delta Q / d B)=-\left.(\partial M / \partial T)\right|_{B}$. Accordingly the zero transitions of the MCE indicate local extrema in magnetization. These extrema can be used as an indicator for the crossover into the Luttinger liquid regime [9].

\section{Hamiltonian, exchange couplings, and theoretical modeling}

As mentioned above, magnetic insulators allow not only a qualitative but also a fully quantitative analysis. The magnetic [13] and thermodynamic [9] properties of the isostructural compound $\left(\mathrm{C}_{5} \mathrm{H}_{12} \mathrm{~N}\right)_{2} \mathrm{CuBr}_{4}$ could be very well described by a minimal spin ladder Hamiltonian:

$$
H=J_{\text {rung }} \sum_{l} \underline{S}_{l, 1} \cdot \underline{S}_{l, 2}+J_{\operatorname{leg}} \sum_{l, k} \underline{S}_{l, k} \cdot \underline{S}_{l+1, k} .
$$

If we apply the same to $\left(\mathrm{C}_{5} \mathrm{H}_{12} \mathrm{~N}\right)_{2} \mathrm{CuCl}_{4}$, we can determine the exchange couplings $J_{\text {rung }} / k_{B}=3.42(7) \mathrm{K}$ and $J_{\text {leg }} / k_{B}=$ 1.34(6) $\mathrm{K}$ from the values of the critical magnetic fields $[12,20]$. Thus we are able to calculate the magnetic and thermodynamic properties by exact diagonalization (ED), the density-matrix renormalization-group (DMRG) method [21-23], and also by mapping of the spin ladder Hamiltonian to the XXZ model. Due to the strong rung coupling, this latter mapping only considers the lowest two rung states and omits the two upper triplet states on a rung. The properties of the XXZ model are obtained within a mean-field theory, which has been shown to be very accurate for these thermodynamic quantities $[24,25]$. The very good agreement between the theoretical results for the spin ladder model and the experiment is evident in Fig. 1. We note that the asymmetry of the experimental data with respect to half magnetization is covered by the full ED calculations, which include all possible high-energy states. This is in contrast to the XXZ model, which is symmetric with respect to half magnetization and therefore also symmetric at the two QCPs. We find our ED calculations in very good agreement with further DMRG calculations, which are not shown in Fig. 1 in order to not overload the figure. 


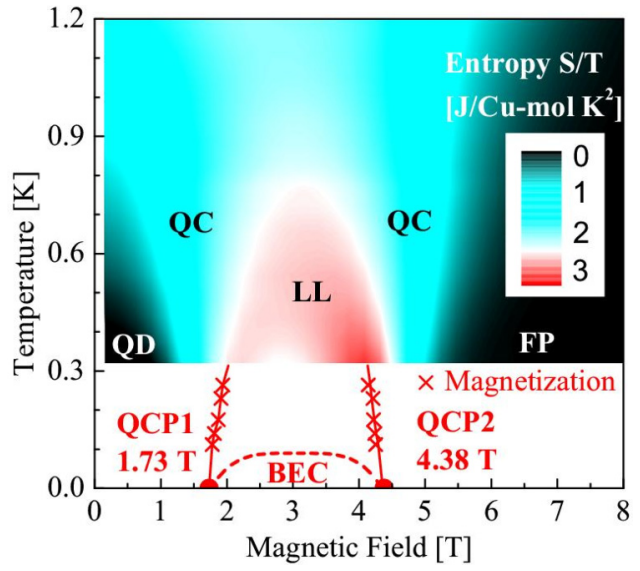

FIG. 2. (Color online) Quantitative entropy landscape $S / T$ of $\left(\mathrm{C}_{5} \mathrm{H}_{12} \mathrm{~N}\right)_{2} \mathrm{CuCl}_{4}$. The typical elements of the spin ladder phase diagram are observed: two QCPs, quantum disordered spin liquid (QD) for $B<B_{c 1}$, spin Luttinger liquid (LL) for $B_{c 1}<B<B_{c 2}$, and full magnetic polarization (FP) for $B_{c 2}<B$. For lower temperatures a Bose-Einstein condensate (BEC) is expected to set in due to the weak coupling between the spin ladders. QC denotes the quantum critical regime.

\section{DISCUSSION}

\section{A. Magnetic entropy}

For further analysis we now calculate the magnetic entropy from the experimental data. The magnetic entropy $S$ is linked to the MCE via

$$
\frac{1}{T} \frac{\delta Q}{d B}=-\frac{d S}{d B}=-\left.\frac{\partial M}{\partial T}\right|_{B} .
$$

Accordingly $S$ can be calculated by magnetic field integration of the MCE data. The fact that entropy is zero at high fields and low temperatures, where all spins are fully polarized, enables the determination of the necessary integration constant. The result at $T=320 \mathrm{mK}$ - gray points in Fig. 1(c)—shows a clear increase of entropy in the critical region due to the continuum of low-lying excitations. Also a good agreement between experiment and ED calculation is observed. Small deviations and slightly negative values near zero field can be attributed to the integration procedure, which causes even slight drifts in the MCE to add up. Figure 2 shows quantitative magnetic entropy data for the whole temperature and magnetic field range. The contour plot resembles the phase diagram of a spin ladder, where the Luttinger liquid regime is visible as an area with high $S / T$, while the quantum disordered spin liquid and the magnetically fully polarized regime show vanishing $S / T$. From Figs. 1(c) and 2 it also becomes clear that entropy accumulates on approaching the QCPs, i.e., two maxima develop, as was theoretically predicted [7]. Figure 2 also shows magnetization data (peaks in $d M / d B$ ), that mark the crossover into the Luttinger liquid down to $100 \mathrm{mK}$. We discuss our entropy data in the context of quantum phase transitions: The concept of QPTs distinguishes two cases, depending on whether long-range order can exist at finite temperature or not. For the first case, a "real" phase transition occurs at finite temperatures upon variation of the control parameter, for example, the magnetic field. Here the QCP can be viewed as the endpoint of this line of finite-temperature transitions [2]. For this case the entropy landscape was experimentally determined by Rost et al. [5]. They studied the metallic oxide $\mathrm{Sr}_{3} \mathrm{Ru}_{2} \mathrm{O}_{7}$, a paramagnet that undergoes a metamagnetic transition near an applied field of $8 \mathrm{~T}$. If no long-range order is possible at finite temperatures, the QCP indicates the crossover from a region in the phase diagram where quantum fluctuations dominate to a region where thermal order parameter fluctuations dominate [2]. This is realized in one-dimensional magnetic insulators with a spin gap like $\left(\mathrm{C}_{5} \mathrm{H}_{12} \mathrm{~N}\right)_{2} \mathrm{CuCl}_{4}$. For this material we are able to identify all phases and show quantitative experimental entropy data, that are in good agreement with theoretical calculations. It is unique so far, because other materials, like $\left(\mathrm{C}_{5} \mathrm{H}_{12} \mathrm{~N}\right)_{2} \mathrm{CuBr}_{4}$, saturating at $14 \mathrm{~T}$, lack the high-field MCE and heat capacity data [9], which are necessary to study the physics at and beyond $B_{c 2}$.

\section{B. Magnetic Grüneisen parameter}

We now turn to the magnetic Grüneisen parameter $\Gamma_{\text {mag }}$, which links the derivative of magnetization with respect to temperature $\partial M / \partial T$ to the magnetic heat capacity $c_{\text {mag }}$ :

$$
\Gamma_{\text {mag }}=-\frac{\partial M / \partial T}{c_{\text {mag }}}=\left.\frac{1}{T} \frac{\partial T}{\partial B}\right|_{s} .
$$

The divergence of $\Gamma_{\text {mag }}$ at a QCP is a very sensitive tool to study quantum phase transitions. This is in contrast to heat capacity and $\partial M / \partial T$, which are suited identifiers of phase transitions at finite temperature but approach zero for $T \rightarrow 0$. Furthermore, a sign change of the Grüneisen parameter occurs close to a QCP [26]. Such behavior was experimentally observed for some materials $[4,27,28]$. Another remarkable feature of $\Gamma_{\mathrm{mag}}$ is that it contains information free of any material-specific parameters, yet it is very challenging to measure, since all data need to be on firm quantitative ground.

Evaluating our heat capacity and MCE data according to Eqs. (2) and (3), we obtain experimental data of $\Gamma_{\text {mag }}$ for the whole magnetic field range and across the two quantum critical regimes, as presented in Fig. 3. For decreasing temperature,

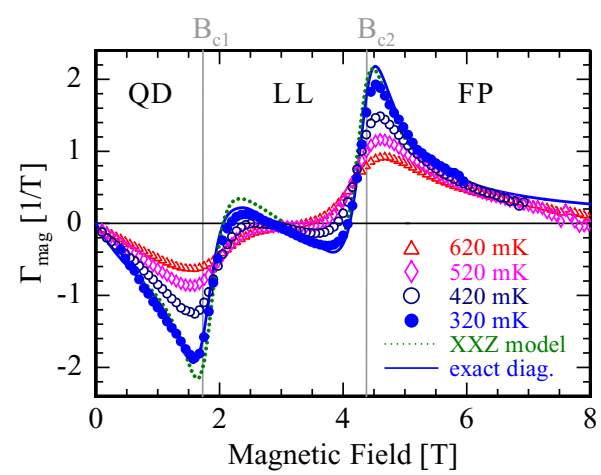

FIG. 3. (Color online) Magnetic Grüneisen parameter $\Gamma_{\text {mag }}$ of $\left(\mathrm{C}_{5} \mathrm{H}_{12} \mathrm{~N}\right)_{2} \mathrm{CuCl}_{4}$ (experimental data) with comparison to calculations: blue line, exact diagonalization 10 rungs; dashed green line, XXZ model. Gray vertical lines mark the critical fields. 


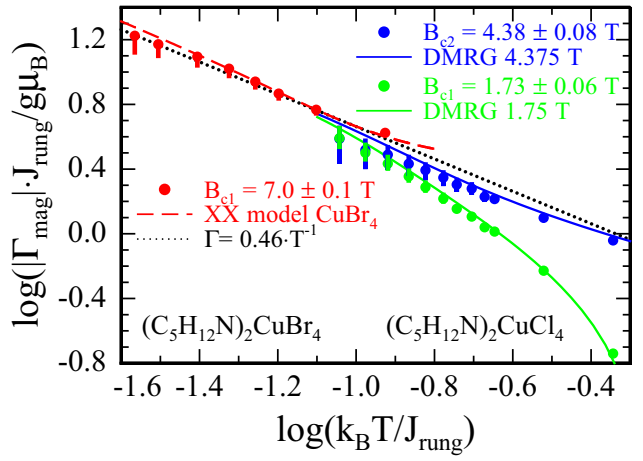

FIG. 4. (Color online) Temperature dependence of $\Gamma_{\text {mag }}$ at the critical magnetic fields, obtained by experiment (points) as well as DMRG (solid lines) and XX chain model (dashed line) calculations for $\left(\mathrm{C}_{5} \mathrm{H}_{12} \mathrm{~N}\right)_{2} \mathrm{CuBr}_{4}$ (left) and $\left(\mathrm{C}_{5} \mathrm{H}_{12} \mathrm{~N}\right)_{2} \mathrm{CuCl}_{4}$ (right). Plotted normalized to the rung coupling $J_{\text {rung }}$ of the two materials $[9,17,19]$.

we observe the predicted sign change and an increase of $\Gamma_{\mathrm{mag}}$ close to the QCPs.

The temperature dependence of $\Gamma_{\mathrm{mag}}$ allows the analysis of the critical behavior for the two QPTs (Fig. 4). Data are available for $\left(\mathrm{C}_{5} \mathrm{H}_{12} \mathrm{~N}\right)_{2} \mathrm{CuCl}_{4}$ for both QCPs and for $\left(\mathrm{C}_{5} \mathrm{H}_{12} \mathrm{~N}\right)_{2} \mathrm{CuBr}_{4}$ for the lower critical field [9]. Since both compounds possess different energy scales, we are able to cover a wide range in energy (temperature). Figure 4 shows the experimental data with respect to $J_{\text {rung }}$ in a double-logarithmic scale in order to demonstrate the occurring power-law behavior. We complement our experimental data by DMRG calculations as well as an analytical approach: For strongly dimerized systems the $S=1 / 2$ ladder can be mapped to the XXZ chain [12,29]. Close to the critical fields there are few excitations or holes, which can be considered noninteracting. This free fermion approximation allows one to map the XXZ chain further to the $\mathrm{XX}$ chain [8]. The advantage of this mapping is that the $\mathrm{XX}$ chain is exactly solvable. Thus, the Grüneisen parameter can be calculated exactly and a scaling $\Gamma_{\text {mag }} \propto T^{-1}$ can be extracted at low temperatures. At the critical field, the XX model is valid for a number of different quasi-one-dimensional (1D) geometries, such as ladders and alternating chains.

In particular, at the different QCPs, the universal scaling $\Gamma_{\text {mag }} \propto T^{-1}$ (dotted line) is consistent with our experimental and theoretical results at low temperature for both compounds and both QCPs, independent of material-specific parameters. For the compound $\left(\mathrm{C}_{5} \mathrm{H}_{12} \mathrm{~N}\right)_{2} \mathrm{CuBr}_{4}$ the experimentally explored temperatures lie fully in the scaling regime $\left(\log \left(k_{B} T / J_{\text {rung }}\right)<-1.0\right)$. In contrast, the $\left(\mathrm{C}_{5} \mathrm{H}_{12} \mathrm{~N}\right)_{2} \mathrm{CuCl}_{4}$ approaches at the lowest measured temperatures the scaling. The very good collapse of the results in Fig. 4 obtained for the two compounds and different theoretical models at low temperatures proves the universal behavior.

Extracting precise critical exponents by directly fitting a power law of the form $a T^{-\alpha}$ to the experimental data is notoriously difficult because of the limited number of data points and the typically unknown extent of the scaling regime. In real materials, the latter may be influenced, e.g., by small additional terms in the Hamiltonian like interladder coupling, as discussed below. Here we profit directly from the guidance provided by the theoretical results in order to determine the scaling regime and to be able to make a meaningful fit. Fitting only the experimental values obtained for the $\mathrm{Br}$ compound we obtain the exponent $\alpha=0.9(1)$, which is close to the theoretical prediction of $\alpha=1$. Analogously, we determine the exponent for the $\mathrm{Cl}$ compound. We use the experimental data at the upper critical field, since here the effects of thermal excitations are smaller. The extracted value is $\alpha \approx 0.9(2)$. A combined fit of both of these data sets results in the exponent $\alpha=1.0(1)$. The good agreement of the DMRG results with all the experimental points gives us the confidence that the theoretical asymptotic exponent 1 is also the asymptotics of the experimental results.

At higher energies (temperatures) the microscopic structure of the material becomes important and deviations from the scaling occur. We observe clearly different behavior for the two QCPs, as seen in the $\left(\mathrm{C}_{5} \mathrm{H}_{12} \mathrm{~N}\right)_{2} \mathrm{CuCl}_{4}$ data. This is due to the energies of the higher triplet excitations which shift with the magnetic field. However, even in this regime, where the details of the microscopic model matter, we find a very good quantitative agreement between experiment and the DMRG calculations for the ladder system (solid lines) within the experimental error bars.

For very low temperatures below $0.03 J_{\text {rung }} / k_{B}$, the experimental data start to deviate from the XX model calculations. We attribute this to the interladder coupling, which will eventually result in a long-range ordered phase between $B_{c 1}$ and $B_{c 2}$ and the occurrence of Bose-Einstein condensation $[10,16,30,31]$. This means that the one-dimensional character is cut off by the proximity of the three-dimensional long-range order and the XX model is no longer valid for this particular compound.

We further find that the critical behavior is very sensitive to changes of the magnetic field. The error bars in Fig. 4 indicate the variation of $\Gamma_{\mathrm{mag}}$ within the uncertainties of the values of $B_{c 1}$ and $B_{c 2}$. At low energies, we note that deviations of the magnetic field toward the Luttinger liquid have a much stronger effect on $\Gamma_{\mathrm{mag}}$ (the downward pointing error bar in Fig. 4) than deviations toward the quantum disordered regime (the upward pointing error bar that is smaller than point size). The very sensitive dependence on the slight changes of the magnetic field was witnessed for the theoretical calculations too.

\section{SUMMARY}

In summary, we presented magnetic and thermodynamic measurements as well as calculations for the two-leg antiferromagnetic $S=1 / 2$ Heisenberg ladder $\left(\mathrm{C}_{5} \mathrm{H}_{12} \mathrm{~N}\right)_{2} \mathrm{CuCl}_{4}$, which features two quantum phase transitions at $B_{c 1}=1.73(6) \mathrm{T}$ and $B_{c 2}=4.38(8) \mathrm{T}$. Using these measurements we are able to give a fully qualitative and quantitative description of magnetic entropy and magnetic Grüneisen parameter in the vicinity of QPTs. We find universal scaling behavior at the two QCPs, are able to extract the critical exponents, and determine the critical regime in temperature and magnetic field. The model character of $\left(\mathrm{C}_{5} \mathrm{H}_{12} \mathrm{~N}\right)_{2} \mathrm{CuCl}_{4}$ allows future studies to gain insight into the thermodynamics of more complicated (disordered) systems. Our study of $\left(\mathrm{C}_{5} \mathrm{H}_{12} \mathrm{~N}\right)_{2} \mathrm{CuCl}_{4}$ paves 
the way for future measurements on samples with different chlorine to bromine ratios to investigate the effects of bond disorder and Bose glasses in 1D antiferromagnets.

\section{ACKNOWLEDGMENT}

This work was supported in part by the Swiss NSF under MaNEP and Division II.
[1] S. Sachdev and B. Keimer, Phys. Today 64, 29 (2011).

[2] M. Vojta, Rep. Prog. Phys. 66, 2069 (2003).

[3] P. Gegenwart, Q. Si, and F. Steglich, Nat. Phys. 4, 186 (2008).

[4] Y. Tokiwa, T. Radu, C. Geibel, F. Steglich, and P. Gegenwart, Phys. Rev. Lett. 102, 066401 (2009).

[5] A. W. Rost, R. S. Perry, J.-F. Mercure, A. P. Mackenzie, and S. A. Grigera, Science 325, 1360 (2009).

[6] T. Tajiri, H. Deguchia, M. Mitoa, S. Takagia, H. Nojirib, T. Kawaec, and K. Takedac, J. Magn. Magn. Mater. 272-276, 1070 (2004).

[7] L. Zhu, M. Garst, A. Rosch, and Q. Si, Phys. Rev. Lett. 91, 066404 (2003).

[8] T. Giamarchi, Quantum Physics in One Dimension (Oxford University Press, New York, 2004).

[9] Ch. Rüegg et al., Phys. Rev. Lett. 101, 247202 (2008).

[10] M. Klanjšek et al., Phys. Rev. Lett. 101, 137207 (2008).

[11] B. Thielemann et al., Phys. Rev. Lett. 102, 107204 (2009).

[12] F. Mila, Eur. Phys. J. B 6, 201 (1998).

[13] B. C. Watson et al., Phys. Rev. Lett. 86, 5168 (2001).

[14] T. Lorenz, O. Heyer, M. Garst, F. Anfuso, A. Rosch, Ch. Rüegg, and K. Krämer, Phys. Rev. Lett. 100, 067208 (2008).

[15] P. Bouillot et al., Phys. Rev. B 83, 054407 (2011).

[16] S. Mukhopadhyay et al., Phys. Rev. Lett. 109, 177206 (2012).
[17] S. Ward, P. Bouillot, H. Ryll, K. Kiefer, K. W. Krämer, Ch. Rüegg, C. Kollath, and T. Giamarchi, J. Phys.: Condens. Matter 25, 014004 (2013).

[18] T. Sakakibara, H. Mitamura, T. Tayama, and H. Amitsuka, Jpn. J. Appl. Phys. 33, 5067 (1994).

[19] B. R. Patyal, B. L. Scott, and R. D. Willett, Phys. Rev. B 41, 1657 (1990).

[20] M. Reigrotzki, H. Tsunetsugu, and T. M. Rice, J. Phys.: Condens. Matter 6, 9235 (1994).

[21] K. A. Hallberg, Adv. Phys. 55, 477 (2006).

[22] E. Jeckelmann, Prog. Theor. Phys. Suppl. 176, 143 (2008).

[23] U. Schollwöck, Ann. Phys. 326, 96 (2011).

[24] F. Heidrich-Meisner, A. Honecker, and W. Brenig, Phys. Rev. B 71, 184415 (2005).

[25] A. V. Sologubenko, T. Lorenz, J. A. Mydosh, B. Thielemann, H. M. Rønnow, Ch. Rüegg, and K. W. Krämer, Phys. Rev. B 80, 220411(R) (2009).

[26] M. Garst and A. Rosch, Phys. Rev. B 72, 205129 (2005).

[27] B. Wolf et al., Proc. Natl. Acad. Sci. USA 108, 6862 (2011).

[28] F. Weickert et al., Phys. Rev. B 85, 184408 (2012).

[29] G. Chaboussant, M.-H. Julien, Y. Fagot-Revurat, M. Hanson, L. P. Lévy, C. Berthier, M. Horvatic, and O. Piovesana, Eur. Phys. J. B 6, 167 (1998).

[30] T. Giamarchi, Ch. Rüegg, and O. Tchernyshyov, Nat. Phys. 4, 198 (2008).

[31] B. Thielemann et al., Phys. Rev. B 79, 020408(R) (2009). 